\title{
Competencias del Profesorado Universitario para la Atención a la Diversidad en la Educación Superior
}

\author{
Competences of University Teaching Staff for Attention to \\ Diversity in Higher Education
}

Eddy Paz Maldonado *

Universidad Nacional Autónoma de Honduras, Honduras

\begin{abstract}
La formación inicial y permanente del profesorado es un factor determinante para desarrollar distintos procesos inclusivos dirigidos a brindar atención a la heterogeneidad de estudiantes en las instituciones de enseñanza superior. Esta diversidad debe ser entendida desde una perspectiva que busque propiciar la creación de un entorno educativo inclusivo. El objetivo es reflexionar sobre las competencias del profesorado universitario para la atención a la diversidad en la educación superior. Revisa numerosas fuentes bibliográficas y propone diez competencias básicas y transversales para abordar a colectivos en condición de vulnerabilidad. El trabajo concluye que el profesorado universitario se enfrenta a un contexto laboral exigente producto de las reformas educativas que se implementan en el funcionamiento de la gestión universitaria. Sin embargo, en la práctica pedagógica han de incorporar mecanismos que permitan la participación, el pluralismo y la democratización de los espacios educativos. Además, deben prepararse constantemente en la utilización de nuevas metodologías de aprendizaje, planificación, mediación y evaluación. En consecuencia, se requieren profesores capacitados, conscientes, comprometidos, con actitud positiva para aceptar y entender las diferencias de los educandos en las universidades. Por esta razón, es necesario que se fortalezcan los programas de profesionalización docente para impulsar nuevas competencias relacionadas con la inclusión y atención a la diversidad en las instituciones de enseñanza universitaria.
\end{abstract}

Descriptores: Competencias del docente; Enseñanza superior; Grupos vulnerables; Igualdad de oportunidades; Práctica pedagógica.

The initial and permanent training of teachers is a determining factor to develop different inclusive processes aimed at providing attention to the heterogeneity of students who participate in higher education institutions. This diversity must be understood from a perspective that search to foster the creation of an inclusive educational environment. The aim is to reflect on the competences of university teaching staff for attention to diversity in higher education. It reviews numerous bibliographical sources and proposes ten basic and transversal competences to address vulnerable groups. The work concludes that the university teaching staff faces a demanding labor context product of the educational reforms that are implemented in the operation of the university management. However, in pedagogical practice they must incorporate mechanisms that allow participation, foment pluralism and the democratization of educational spaces. In addition, they must prepare constantly in the use of new learning methodologies, planning, mediation and evaluation. Consequently, trained, conscious, committed teachers with a positive attitude are required to accept and understand the differences of the students in the universities. For this reason, it is necessary to strengthen teacher professionalization programs to impulse new competencies related to inclusion and attention to diversity in the university education institutions.

Keywords: Teacher qualifications; Higher education; Vulnerable groups; Equal opportunity; Pedagogical practice.

*Contacto: eddy.paz@unah.edu.hn

ISSN: 0718-7378

www.rinace.net/rlei/
Recibido: $\quad 17 / 04 / 2018$

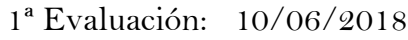

Aceptado: 07/08/2018 


\section{Introducción}

En los centros de educación superior participan estudiantes de diferentes colectivos, con características y necesidades particulares. Para lograr la cohesión socioeducativa en dichos centros, se tiene que prestar atención de forma efectiva a todos estos educandos. En los últimos años se han efectuado reformas universitarias que proponen una serie de modificaciones para brindar respuesta a la diversidad. Sin embargo, el profesorado no se encuentra preparado para atender a grupos heterogéneos e implementar las medidas establecidas en las instituciones educativas. Por esta razón, la atención a la diversidad constituye un gran desafío para los educadores hoy en día (Murillo et al., 2010).

Existen distintas concepciones sobre la diversidad. Cárdenas (2012) dice que este término es muy utilizado en el contexto educativo actual. Arnaiz (2003, p. 15) declara la diversidad "como el conjunto de características que hacen a las personas y a los grupos distintos con relación a factores físicos, genéticos, culturales y personales”.

Según Parilla (1999), la diversidad hace referencia a variados colectivos heterogéneos, a multiplicidad de representaciones o manifestaciones dentro de esos grupos, sin importar la fuente de esa heterogeneidad. Por tanto, existe diversidad en un colectivo en razón de edad, intereses, religión, capacidad mental, color de piel, sexo, o procedencia social de sus miembros. Rosales (2012) coincide que el origen étnico, cultura, clase social, género, religión, ideología política, diferentes capacidades y necesidades son elementos a tomar en cuenta para analizar la heterogeneidad en los estudiantes de los centros de enseñanza superior.

Álvarez (2018) manifiesta que el profesorado tiene que aceptar y respetar la diversidad de forma genuina. También, aprender a valorarla como una nueva oportunidad de aprendizaje y no como un problema. Más allá de los discursos, la idea de que la diversidad es vida, de que el estudiantado es distinto y deben respetarse todas esas particularidades, ha de estar firme y auténticamente adherida en todos los docentes.

La educación superior, tiene la obligación de brindar propuestas para la solución de problemáticas relacionadas con el acceso, egreso y permanencia del estudiantado. Las instituciones en este nivel, necesitan adaptarse a modalidades de formación más coherentes con la realidad social. Por ejemplo, mejorar las aulas regulares, implementar nuevas metodologías de aprendizaje y diseñar otras formas de evaluación para construir mejores espacios educativos (Salinas, de Benito y Lizana, 2014). Se demanda un profesorado con una serie de competencias para ofrecer una educación de calidad. Es necesario diseñar un perfil docente que integre diferentes competencias, que faciliten un mejor desempeño de las funciones de los académicos en las universidades (Mas y Olmos, 2016).

Este artículo tiene como objetivo reflexionar acerca de las competencias del profesorado universitario para la atención a la diversidad en la educación superior. Revisa numerosas fuentes bibliográficas sobre el tipo de competencias que deben poseer y desarrollar los docentes para abordar a estudiantes en situación de discapacidad, pueblos indígenas, afrodescendientes, diversidad sexual e inmigrantes. Para cumplir con los propósitos planteados, el trabajo se estructura en cuatro secciones: en la primera, se expone la atención a la diversidad en la educación superior; en la segunda, se describe la formación del profesorado para la atención a la diversidad en la enseñanza superior; en la tercera, se analizan diferentes perspectivas sobre las competencias del profesorado universitario 
para el ejercicio docente y la atención a la diversidad. Finalmente, se presenta una propuesta sobre las competencias básicas y transversales para atender a la diversidad en la educación superior.

\section{La atención a la diversidad en el contexto de la educación superior}

Atender y entender las diferencias del estudiantado es uno de los grandes retos que se presentan actualmente en la educación superior. Para ello, se requieren mecanismos funcionales que permitan construir una universidad abierta y pluralista. Una universidad democrática que admita los planteamientos de los educandos en la búsqueda por lograr la participación de toda la comunidad universitaria. La atención a la diversidad es una acción determinante para construir centros de enseñanza superior inclusivos, que posibiliten el desarrollo de los múltiples grupos marginados y excluidos socialmente.

La diversidad es un fenómeno visible en las universidades del sistema de educación superior, pero la misma no ha sido atendida de la mejor manera. La formación inicial docente que brindan los centros de enseñanza superior carece de un modelo auténtico, apropiado, congruente y sensible a las características de los educandos. Por otra parte, los contenidos referentes a la educación inclusiva y atención a la diversidad se presentan como asignaturas optativas y no indispensables, lo que produce una base débil para abordar dicha temática por parte de los educadores (Domínguez y Vázquez, 2015).

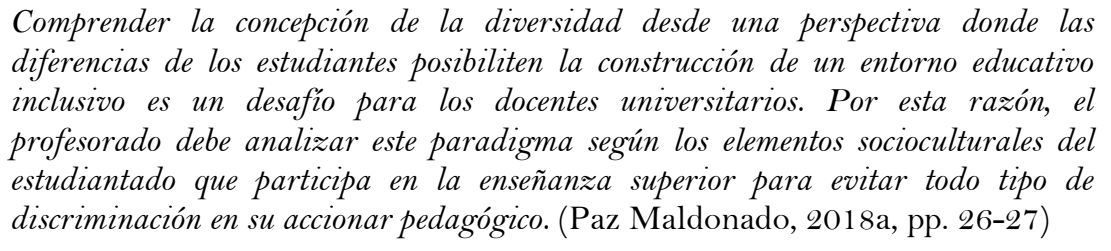

Los términos equidad, atención a la diversidad e inclusión educativa se encuentran inmersos en la discusión pública referente a la educación, distintos autores los exponen como iguales, para Herrera-Seda, Pérez-Salas y Echeita (2016) son elementos complementarios para el reconocimiento y valoración de la diversidad estudiantil en las prácticas pedagógicas, y políticas educativas.

Según Booth y Ainscow (2015), la diversidad toma en consideración las diferencias evidentes, no palpables y las semejanzas entre los sujetos. Esta diversidad trata de la desemejanza dentro de una sociedad común y engloba a todos los individuos sin importar su condición. No obstante, la valoración de la diversidad tiene sus limitaciones. Cuando no se acepta a los demás como son, provoca el no reconocimiento y la negación de nosotros mismos.

Para entender la diversidad en la educación superior es importante considerar el tipo de sociedad en el que nos desarrollamos. Una sociedad donde predomina la segmentación de diversos grupos y donde las diferencias humanas son consideradas como un problema, y no como una oportunidad para lograr evitar la exclusión. Esta diversidad se encuentra íntimamente relacionada con el contexto social, político, económico y personal, no solo educativo (López-Melero, 2012).

La atención a la diversidad es una temática que requiere la formación del profesorado bajo el enfoque de justicia social. Un enfoque desatendido en los programas formativos 
de los futuros y actuales educadores. Según Silva-Peña (2017, p. 126), "el concepto de Justicia Social podemos comprenderlo de un modo general como el camino teóricopráctico que se ha emprendido para luchar contra las desigualdades”. Además, Zeichner (2017) menciona que la justicia social se orienta a formar docentes dispuestos y capaces de trabajar en diversos contextos educativos con el fin de hacerle frente a las desigualdades que existen en la enseñanza y en la sociedad. También, reconoce los aportes de los educadores para acrecentar las oportunidades de vida del estudiantado.

La atención a la diversidad es una de las exigencias que la sociedad y diferentes legislaciones hacen a los establecimientos de enseñanza superior. Las universidades deben atender a todo el estudiantado, sin importar sus características o condiciones personales. Esta atención tiene que ser de calidad y brindar oportunidades iguales en la vida universitaria. En el contexto académico debe ser el profesorado el que atienda los requerimientos que puedan presentar los educandos. Por tanto, estos docentes tienen que desarrollar competencias relacionadas con la atención a distintos grupos, para ofrecer una formación adecuada a los estudiantes que tienen a su cargo (Díez y Sánchez, 2015).

La historia de la diversidad pasa por etapas que van desde la aceptación de que todo el estudiantado tiene derecho a recibir educación, hasta la implementación de acciones que busquen ofrecer una enseñanza de calidad a todos los educandos en los espacios áulicos regulares (García et al., 2013).

Jiménez y Fardella (2015) mencionan que existen diferentes formas de valorar la diversidad en la educación. En primer lugar, la negación de la diversidad que se caracteriza por invisibilizar las diferencias de origen sociocultural y la diversidad como problema, esta se relaciona con las situaciones de incertidumbre que se originan dentro de las aulas de clase y que el profesorado no se encuentra preparado o dispuesto a atender.

Hablar de la diversidad del estudiantado en términos de aprendizaje, es referirnos a que quien aprende no es un grupo sino cada sujeto y cada uno de ellos a su manera. Una docencia basada en el aprendizaje de nuestros estudiantes nos obliga a estar pendientes de cada uno de ellos, a supervisar el proceso que van a seguir, a facilitar su progreso a través de los dispositivos didácticos cuyo dominio se nos supone como profesionales de la enseñanza, en definitiva, una enseñanza más personalizada. (Fernández Batanero, 2013, p. 84)

La atención a la diversidad en la enseñanza superior requiere desarrollar múltiples acciones por parte de toda la comunidad educativa. Sin embargo, el profesorado tiene una enorme obligación en la búsqueda por ofrecer una educación inclusiva que permita fomentar el pluralismo sin ninguna distinción en todos los centros educativos de este nivel, dado que, son ellos los que diariamente interactúan con el estudiantado en los espacios de formación universitaria.

\section{La formación del profesorado para la atención a la diversidad en la enseñanza superior}

La carrera docente a mediados del siglo diecinueve surge con la idea de que los profesionales que se dedicarían a la labor de educar tendrían que ser personas con un alto grado de vocación, dedicación, sacrificio para ejercer la misma y mantenerse en la 
profesión durante toda la vida académica. En el siglo veinte la figura del educador está ligada al aspecto técnico. Además, presenta mayor capacidad para tomar decisiones respecto a los procesos de enseñanza-aprendizaje. La tarea del profesorado es de servicio, pero participa de manera activa al compartir sus conocimientos con pares, capacitándose a diario ante los nuevos desafíos que surgen producto de las transformaciones socioeducativas (Veloquio, 2016).

\begin{abstract}
Las universidades históricamente han respondido a un paradigma academicista donde el énfasis ha estado puesto en la transmisión de conocimientos, centrado en una fuerte fragmentación disciplinar. Desde ahi las instituciones de educación superior se han constituido como agencias transmisoras de la cultura dominante, asumiendo el poder y la regulación respecto a cuál es el conocimiento valido, y quienes pueden acceder a él. Por su parte, el actual modelo profesionalizante que ha ido instalándose en los procesos de renovación curricular de las universidades fortalece un marco de competencias tendientes a homogenizar los desempeños profesionales. (Schilling, 2018, p. 150)
\end{abstract}

En América Latina, la función del profesorado se encuentra en una etapa de intensas transformaciones relacionadas con las exigencias provocadas en la sociedad; entre estas, reconocer la diversidad desde un sentido amplio como ser cultural, migratorio, de género, funcional, entre otras y la petición por parte de la población para asumir un papel relevante en el desarrollo social. No obstante, lo expuesto anteriormente repercute de manera directa en los sistemas educativos, principalmente en el ejercicio docente y en las universidades formadoras de educadores. Los centros de enseñanza superior producto de las reformas educativas constantes han creado modelos que no ofrecen respuestas contundentes para atender a la diversidad en todas sus formas (Ferrada, 2017).

Para que las y los educadores se conviertan en personas capaces de modificar los centros de enseñanza tradicional en inclusivos, deberán transformarse los sistemas de formación docente. La formación inicial es la primera etapa en la que los futuros educadores obtienen las bases necesarias para entender su rol mediante el desarrollo de competencias relacionadas con la diversidad en la educación. También, la formación permanente debe prepararles para implementar medidas que solucionen la problemática de atender a la heterogeneidad en la educación. Por tanto, es necesario un nuevo perfil profesional para formar docentes reflexivos y críticos ante las diversidades que se dan en los contextos educativos actuales (Domínguez y Vázquez, 2015).

La formación inicial docente es fundamental en la búsqueda del respeto a la diversidad y el logro de espacios áulicos inclusivos, debido a que en esta etapa se promueven aspectos actitudinales, creencias y se desarrollan múltiples concepciones en base a la educación, que serán guía para el ejercicio de la profesión en los sistemas educativos. Por eso, es vital el análisis de la perspectiva relacionada con la inclusión educativa para atender a la diversidad en las universidades que forman al futuro profesorado. Lo que sería de mucha importancia para comprender la idea que los docentes conocen y desarrollan en su práctica pedagógica sobre dicha perspectiva (Vélez et al., 2016).

Para que se instaure la educación inclusiva y se brinde respuesta inmediata a la diversidad, es esencial modificar la formación de los futuros educadores. Los establecimientos de enseñanza solo podrán realizar esas reformas si son conscientes de la importancia de ese aspecto (García y Arroyo, 2014). Además, debe construirse una propia identidad en el transcurso de la carrera docente por parte del profesorado, debido a que hoy en día tendrán que revisar constantemente su práctica pedagógica para 
adquirir y desarrollar competencias a través de la formación permanente en el propio entorno laboral donde se desenvuelven (Matus, 2013).

Los centros de enseñanza superior necesitan implementar diferentes mecanismos para promover la participación de toda la comunidad educativa y desarrollar procesos de atención a la diversidad, desde un enfoque más amplio para evitar el asistencialismo a colectivos más visibles socialmente como, por ejemplo, el estudiantado en situación de discapacidad. La creación de programas de formación docente es un elemento fundamental para contribuir con la generación de competencias básicas y transversales que permitan entender y atender la misma (Paz Maldonado, 2018b).

Uno de los grandes desafíos que enfrenta la formación inicial docente es la preparación de educadores conscientes de sus propios prejuicios y capacidades para enseñar en contextos de alta inestabilidad. La universidad se convierte en un espacio donde los futuros docentes pueden reconocer y cambiar sus propios prejuicios, así como reflexionar sobre los mismos al momento de ejercer su profesión (Salas Guzmán y Salas Guzmán, 2016). El reconocimiento de la diversidad exige la transformación de los procesos educativos actuales (Guzmán Marín, 2018).

El profesorado que ingresa al sistema educativo debe tener vocación para desempeñarse en sus diferentes instituciones. No puede ser insensible ante la realidad política, económica, social y educativa que existe en estos tiempos. Por ello, los centros de formación docente tienen una enorme responsabilidad en la construcción de una sociedad más inclusiva. Freire (1993) afirma que quien pretende enseñar tiene que desarrollar cualidades relacionadas con la humildad, amorosidad, valentía, tolerancia, capacidad de decisión y fomento de la democracia.

\section{Competencias del profesorado universitario para el ejercicio docente y la atención a la diversidad}

En este apartado se plantean algunas distinciones teóricas sobre las diferentes perspectivas de concebir las competencias del profesorado para el ejercicio docente y la atención a la diversidad en la educación superior.

La enseñanza universitaria solicita un conjunto de habilidades y competencias por parte del profesorado. En las instituciones de educación superior se exige que el docente incluya dentro de su práctica educativa la investigación, la implementación de metodologías apropiadas para lograr el desarrollo del estudiantado y la búsqueda por resolver una serie de contrastes que respondan a las necesidades requeridas por los mismos (Rivadeneira, 2017).

Las competencias del profesorado en la enseñanza superior incluyen todos los elementos relacionados con su práctica pedagógica, con su finalidad, con la preocupación por mejorarla y con su profesionalización. Por ende, para comprenderlas es importante considerar tres aspectos: el contenido, la clasificación y la formación, es decir, saber que enseñar, cómo enseñar, a quienes se enseña y para qué (Torres et al., 2014). Rey (1999) expone que:

Existen dos modelos opuestos de competencia. En el primero, la competencia se encuentra ligada al cumplimiento de una función que corresponde a una clase de situaciones y aun cuando no se reduce a un comportamiento, como en la forma límite 


\begin{abstract}
e insostenible se puede describir como una organización de comportamientos. En ese sentido es particularmente específica. El otro modelo la concibe como una capacidad generativa susceptible de engendrar una infinidad de conductas adecuadas respecto a una inmensidad de situaciones nuevas. (p. 33)
\end{abstract}

Según Zabalza (2003), las competencias docentes, son el conjunto de conocimientos y habilidades que los sujetos necesitamos para desarrollar algún tipo de actividad en la vida cotidiana. Larraín y González (2005) afirman que son cualidades de los seres humanos que contribuyen a desarrollar un mejor trabajo. Le Boterf (2001) define la competencia como "un saber actuar responsable y validado, que combina diferentes recursos endógenos (capacidades, aptitudes, formación, experiencia) y exógenos (redes de comunicación, de documentación de expertos y de herramientas)" (p. 53). Para Perrenound (2003), "la competencia es la capacidad de actuar eficazmente en una situación dada, capacidad que se fundamenta en algún conocimiento, pero que no se reduce a él” (p. 57).

Las competencias han modificado las fases de formación del profesorado, su introducción al mundo profesional, su crecimiento profesional permanente y valoración de su propia práctica pedagógica (Danielson, 2011). Según Valcárcel, Quintana y Oramas (2014) este modelo de competencias requiere de un desempeño profesional pedagógico productivo para satisfacer las necesidades de la gestión educativa en los diferentes espacios universitarios y las exigencias sociales de la actualidad.

\begin{abstract}
La competencia profesional del docente, entendida más como una competencia intelectual, trasciende el sentido puramente técnico del recurso didáctico. Las competencias docentes se caracterizan por ser complejas: combinan habilidades, principios y conciencia del sentido y de las consecuencias de las prácticas pedagógicas; así como una reflexión y análisis sobre los contextos que las condicionan y que van más allá del aula. (Barrón, 2009, p. 78)
\end{abstract}

Zabalza (2009) considera que todo profesor universitario debe: planificar el proceso de enseñanza-aprendizaje, seleccionar los contenidos disciplinares, ofrecer explicaciones comprensibles a sus estudiantes, manejar didácticamente las nuevas tecnologías, gestionar metodologías de trabajo didáctico, relacionarse constructivamente con los educandos, Tutorizar al estudiantado y profesorado, evaluar los aprendizajes, reflexionar e investigar sobre la enseñanza y finalmente, implicarse institucionalmente.

Por otra parte, diferentes estudios hacen énfasis en las competencias didácticas. Por ejemplo, Rivadeneira (2017) afirma que el profesorado universitario asumirá un nuevo rol de acompañante, facilitador, guía, orientador de un proceso de conocimiento, capaz de estimular cada vez más el desarrollo individual del estudiantado, con responsabilidad y autonomía para su perfeccionamiento. También, Valcárcel, Quintana y Oramas (2014) coinciden en que los modelos de competencias docentes deben estar centrados en los educandos y el profesorado deberá buscar desarrollar una postura de estimular el aprendizaje, ser un tutor y contribuir a que los estudiantes sean mejores aprendices en el proceso educativo.

Para dar respuesta a los cambios culturales, políticos, sociales y económicos al profesorado universitario se le exige el dominio de idiomas, manejo de la informática, desarrollar competencias interactivas, comunicacionales o socio-relacionales, el manejo de relaciones humanas, flexibilidad, polivalencia y versatilidad (Barrón, 2009). En cambio, Más y Olmos (2016) plantean que estos cambios y la repercusión de los mismos en la educación superior provocan alteraciones en las funciones del profesorado, por lo 
que es necesario delimitar un nuevo perfil y conocer las competencias pedagógicas ya establecidas. Tobar (2017) resalta la importancia de la dimensión pedagógica en el índice de competencias docentes de educación superior para fortalecer las competencias TIC en busca de desarrollar la investigación y gestión educativa para beneficiar a los educandos.

Por otro lado, Villarroel y Bruna (2014) analizan la incorporación de las competencias genéricas en las mallas curriculares para la formación integral de los estudiantes, pero afirman que, se requiere que los profesores posean las mismas para lograr promoverlas en su accionar pedagógico. Por ello, la formación de los académicos es fundamental para las mejoras educativas. Zabalza (2012) sugiere que es importante visibilizar el proceso de construcción de propuestas curriculares basadas en competencias como marco de referencia y como elemento de contraste en relación a la viabilidad del enfoque formativo inicial de profesores.

En la educación superior, participan activamente estudiantes de diversos colectivos que forman parte de la heterogeneidad universitaria, entre ellos: en situación de discapacidad transitoria y permanente, pueblos indígenas, afrodescendientes, diversidad de género, diversidad sexual, diversidad cultural, estudiantes extranjeros e inmigrantes, por ello, los académicos deben ser formados sólidamente para entender y atender la diversidad en los distintos espacios de aprendizaje. Es vital poder desarrollar una educación bajo el enfoque intercultural. Para García y Jáuregui (2013), la interculturalidad es una acción que se opone a todo tipo de prácticas segregadoras, dado que, se limita a los fundamentalismos ideológicos, la discriminación y propone disminuir la desigualdad social a través del diálogo y el juicio crítico.

Los profesores universitarios deben poseer una serie de características profesionales para posibilitar una enseñanza basada en el respeto a las diferencias de todo el estudiantado. En ese sentido, para Amaro, Méndez y Mendoza (2014), la comunicación con los estudiantes, la motivación desde factores contextuales, la tutoría integrada desde la docencia, la práctica reflexiva, la gestión del aprendizaje, la promoción del aprendizaje colaborativo, la planificación basada en las diferencias, la colaboración con otros profesores para la construcción de estrategias de aprendizaje y la mejora sostenida de la práctica, son elementos que contribuyen en gran medida para atender a los educandos de diversos grupos sociales.

En el caso del estudiantado con necesidades educativas específicas, Álvarez-Pérez y López-Aguilar (2015) dicen que el profesorado universitario debe estar preparado para realizar diversas tareas. Es decir, ayudar al estudiante a integrarse a la vida universitaria, atender las necesidades académicas, dar respuestas en el aula a los requerimientos que presenten los mismos y realizar intervenciones tutoriales para facilitar su aprendizaje.

Para entender la diversidad cultural es importante que los docentes tengan conocimiento acerca de diferentes perspectivas y se desenvuelvan de manera respetuosa referente a las características humanas que hacen a las personas diferentes unas de otras. Por ejemplo, edad, raza, grupo étnico, género, habilidades, cualidades físicas, orientación sexual, educación, estado civil y creencias religiosas (Duran y Parra, 2014).

Evitar la discriminación en base a orientación sexual y género, demanda la sensibilización de los educadores para la introducción en el currículo de estos tópicos. Los docentes deben proporcionar información y servicios de apoyo al estudiantado sobre 
dichas temáticas (Martxueta y Etxeberria, 2014). Para entender y respetar la diversidad sexual es necesario que el profesorado elimine toda clase de estigmas referentes a la identidad de género y orientación sexual de las personas que pertenecen a este colectivo. "Los prejuicios religiosos, políticos y sociales limitan la promoción de la tolerancia y participación en los espacios de aprendizaje" (Paz Maldonado, 2018a, p. 26).

Santos, Cernadas y Lorenzo (2014) dicen que los profesores deben ser formados para desarrollar el dominio de técnicas de aprendizaje cooperativo para fomentar una educación pluralista que permita la cohesión de los educandos.

En lo concerniente a, los aspectos fundamentales para la creación de espacios áulicos democráticos, la actitud del profesorado es un elemento clave para respetar las diferencias, y atender la diversidad del estudiantado en las aulas de clase en la educación superior (Rodríguez, Álvarez y García-Ruiz, 2014). Para comprender mejor los escenarios de aprendizaje y sus implicaciones Salinas, de Benito y Lizana, (2014), señalan que el docente requiere de competencias para desarrollar nuevas formas de trabajo. Es decir, las competencias tecnológicas y sobre todo las comunicativas para promover la adquisición de habilidades en los educandos.

Según Eslava, de León y González (2015), es indispensable el desarrollo de competencias transversales tales como: trabajo en equipo, participación activa, autonomía personal y emprendimiento para atender a la diversidad. Por su parte, Fernández Batanero (2013), expone que el profesorado universitario debe tutorizar al estudiantado, propiciar el diálogo, gestionar metodologías activas, adecuar la enseñanza y los materiales educativos, y colaborar con todos los agentes educativos.

Profundizar sobre la enseñanza es un aspecto clave para entender la diversidad en la educación. Sin embargo, la función docente no solo está ligada al aspecto pedagógico, sino que los educadores deben presentar sensibilidad ante los estudiantes para generar un aprendizaje reflexivo, significativo y crítico que les permita analizar las dimensiones sociales donde se desenvuelven (Cañedo y Figueroa, 2013). Las competencias docentes concretas, deben ser parte de la formación académica de todo profesor. No importa que algunos educadores tengan que atender a individuos en condiciones particulares, estas son de base común para todo el profesorado que se desempeña en la enseñanza superior de cualquier entorno.

El profesorado universitario debe ser capaz de saber escuchar, separar sus propios esquemas culturales, ser empático, evitar los juicios, las críticas y acoplarse con las necesidades, intereses y sentimientos de los educandos, más cuando estos provienen de un contexto cultural distinto (Álvarez, 2018).

Fernández Batanero (2012), señala que el profesorado para generar la inclusión educativa y atender a la diversidad en los espacios universitarios debe poseer y desarrollar competencias pedagógico-didácticas, de liderazgo, gestionar al grupo y al aprendizaje cooperativo, competencias investigativas, interactivas, éticas, sociales, y organizativas.

Es necesario que el cuerpo docente posea la formación que permita conocer y aplicar estrategias de inclusión y diversidad. Para ello, las facultades formadoras de educadores y educadoras, en sus distintos niveles y carreras, deben evidenciar compromiso y conocimiento teórico y práctico, desde una mirada que valore la diversidad (como parte de su perfil de egreso), acompañado de acciones concretas, plasmadas en sus mallas curriculares de formación de docentes, donde la diversidad 
sea un recurso de apoyo al aprendizaje, y no un obstáculo o déficit a subsanar.

(Salas Guzmán y Salas Guzmán, 2016, p. 82)

El profesorado competente es aquel que en su ejercicio profesional es capaz de poner en práctica una serie de conocimientos, destrezas y actitudes para poder solucionar diversas situaciones en el entorno educativo. Por tanto, la formación de competencias debería impulsarse en la formación inicial y permanente de los educadores (Aguaded, de la Rubia y González, 2013).

Según Olmos, Romo y Arias (2016) una de las mayores demandas en la universidad es la atención a la diversidad estudiantil y de la cultura académica. El diálogo y la enseñanza recíproca entre colegas mediante las narrativas pedagógicas de vida, pueden favorecer la construcción de una cultura incluyente, que no disminuya los contextos donde se desempeña el estudiantado, ni las relaciones sociales y pedagógicas que puedan restringir o apoyar una educación de calidad para todos, a través de una pedagogía para la diferencia que reconoce y permite visibilizar a los colectivos que históricamente han sido marginados.

\section{Una propuesta sobre competencias del profesorado universitario para atender la diversidad}

En esta sección se propone una serie de competencias básicas y transversales que los programas universitarios de formación inicial y permanente del profesorado, deberían considerar para la atención a diversos colectivos en condición de vulnerabilidad en las instituciones de educación superior, específicamente los estudiantes en situación de discapacidad, pueblos indígenas, afrodescendientes, diversidad sexual e inmigrantes.

Planificar el proceso educativo en base a las necesidades del estudiantado. Es fundamental que el accionar educativo se dirija a través de una planificación coherente, flexible y que potencie el desarrollo de todos los educandos.

Implementar metodologías que promuevan el respeto, cooperativismo y eviten la discriminación. Este tipo de mecanismos contribuirán a incentivar un posicionamiento positivo por parte de los estudiantes y docentes sobre la diversidad de colectivos que participan a diario en las aulas universitarias, lo que facilitará el trabajo en equipo y la construcción de una visión humanista.

Evaluar de acuerdo a las condiciones de cada estudiante. La evaluación, debe considerar las diferencias de cada educando. Por ejemplo, las personas en situación de discapacidad requieren de ciertos apoyos. Otro caso, son los estudiantes pertenecientes a pueblos indígenas y afrodescendientes, que en ocasiones solo hablan su lengua materna, por lo que, es necesario pensar que tipo de herramientas se utilizarán para evaluar en los distintos espacios educativos. Es esencial la implementación de diversas formas de evaluación de acuerdo a la disciplina en que los docentes se desempeñen. Los ensayos, estudios de caso, resolución de problemas, evaluación de proyectos, debates grupales, conversatorios, pruebas escritas y exámenes orales, son solo algunas formas de evaluar los contenidos desarrollados.

Capacidad comunicativa. Las ideas y opiniones por parte del profesorado, deben dirigirse de forma clara y precisa. No debe utilizarse terminología peyorativa ni excluyente al momento de impartir nuestra práctica pedagógica. En el caso del estudiantado en 
situación de discapacidad visual, debe describirse toda la información proyectada, como ser: gráficos, cuadros y tablas, para que exista una mejor explicación de la temática que se imparte.

Efectuar innovaciones tecnológicas. Estas innovaciones se implementan con el objetivo de evitar la inequidad de accesos y potenciar los espacios educativos. Su uso tiene que realizarse con un propósito preciso para los educandos y el profesorado. Sin embargo, para desarrollar estas innovaciones es necesario considerar si el estudiantado se encuentra en condiciones para realizar las mismas.

Renovar constantemente la práctica pedagógica. La capacitación, permite la actualización continua por parte del profesorado. No obstante, estos deben ser comprometidos y gestionar diferentes espacios de formación permanente relacionada con la atención a distintos grupos vulnerables. La práctica pedagógica constituye el momento preciso para fomentar la inclusión educativa.

Considerar las diferencias del estudiantado. Cada estudiante presenta características particulares. El profesorado universitario debe tomar en cuenta las diferencias como elementos positivos, en la búsqueda de lograr la cohesión pluralista en las universidades.

Conciencia y sensibilidad social. Estas características permiten repensar el aprendizaje desde diversos panoramas, pero se debe considerar la realidad de los sujetos que participan en la educación superior. El rol de acompañante por parte del profesorado, facilita la construcción de nuevos puntos de encuentro, entorno a ideas y pensamientos expresados por los educandos. Los académicos, tienen que ser sensibles ante los problemas sociales y educativos que se viven hoy en día en las distintas sociedades.

Promover la participación del estudiantado en los diferentes espacios de aprendizaje. Los docentes deben ser capaces de respetar las ideas de los educandos. Además, garantizar sus derechos en las instituciones de enseñanza superior, con el fin de democratizar los entornos de aprendizaje. El profesorado debería valorar y respaldar toda acción de los estudiantes en la búsqueda por la mejora de la calidad educativa, puesto que, ellos son el motor que le da vida a las universidades.

Pensamiento reflexivo y crítico. Los estigmas con base en las creencias radicales de origen ideológico, religioso y político, son factores que en el ejercicio docente no deben existir. Para entender la diversidad en la educación superior, es necesario evitar todo tipo de prejuicios. Se requiere un profesorado consciente de las diferencias, con un pensamiento crítico y reflexivo de las distintas realidades educativas, y que visualice desde una nueva perspectiva el proceso educativo. Es importante realizar periódicamente una autoevaluación sobre el ejercicio profesional para conocer las fortalezas y debilidades. En la figura 1 se presentan las competencias básicas y transversales para atender a la diversidad en las instituciones de educación superior.

\section{Conclusiones}

Este artículo señala que las competencias docentes para la atención a la diversidad deben ser incluidas en los programas de formación inicial y permanente del profesorado que se desempeña en la educación superior. Sin embargo, la formación inicial constituye una etapa determinante para la adquisición de las mismas. 


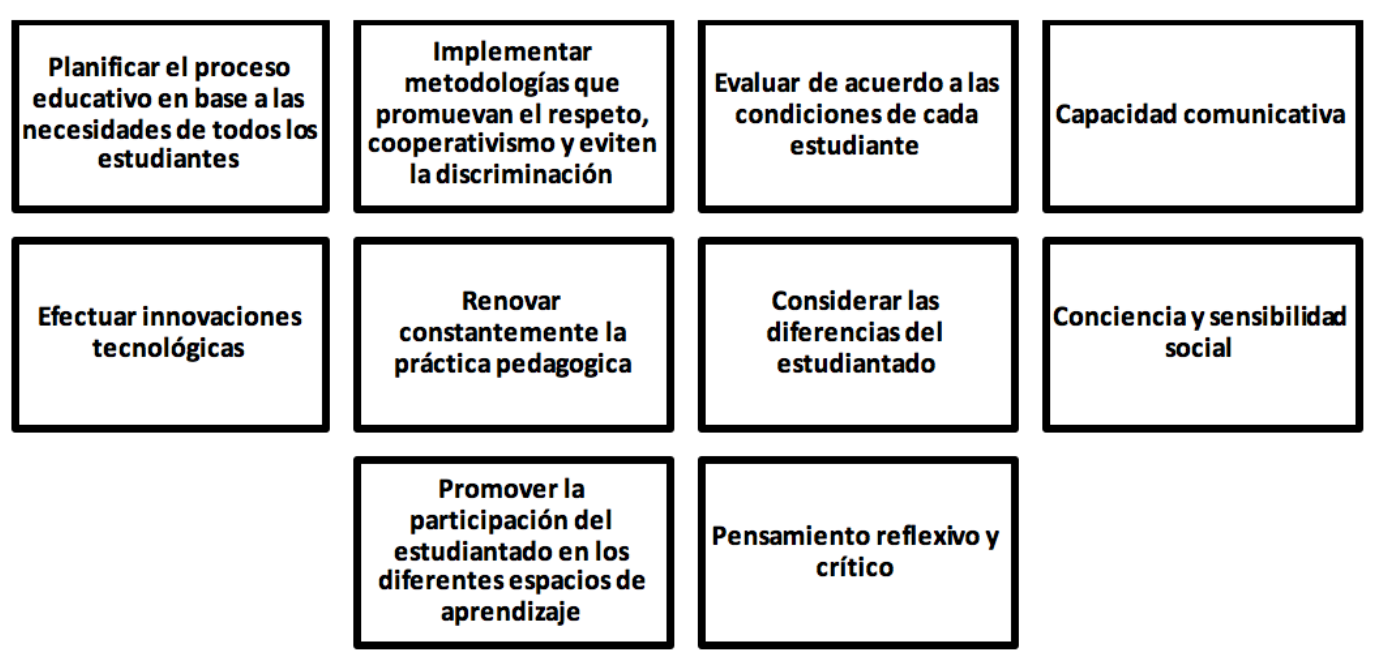

Figura 1. Propuesta sobre las competencias básicas y transversales para atender a la diversidad en la educación superior

Fuente: Elaboración propia.

Las competencias del profesorado universitario para la atención a la diversidad cambian de acuerdo al tipo de estudiantes que sean abordados, políticas públicas y el contexto geográfico donde desarrollen las prácticas pedagógicas los académicos. Por tanto, las investigaciones exploradas plantean que el profesorado universitario debe poder desarrollar competencias didácticas, pedagógicas y genéricas para desenvolverse en la educación superior. Asimismo, diversos estudios afirman que los docentes tienen que formarse y perfeccionar las competencias pedagógicas, de planificación, metodológicas, de evaluación, investigación y gestión. Estas capacidades se complementan junto con las competencias personales y de formación ciudadana.

Las competencias transversales son las que contribuyen más a generar transformaciones actitudinales para entender y atender al estudiantado. La diversidad puede ser considerada desde múltiples perspectivas, por ejemplo, cultural, física, ideológica, religiosa, racial y política.

Para la atención a la diversidad es necesaria la formación y desarrollo de competencias genéricas o específicas para atender a diferentes colectivos que participan en el proceso de enseñanza universitaria. Por ejemplo, la comunicación, motivación, promoción del aprendizaje colaborativo y planificación basada en las diferencias. También, la conciencia social es un elemento inherente que los docentes deben poseer para hacer frente a las distintas problemáticas sociales y en el contexto de enseñanza. En diferentes establecimientos de educación superior de América Latina, se carece de programas de atención a estudiantes en situación de discapacidad, pueblos indígenas, afrodescendientes, diversidad sexual e inmigrantes. Es un primer desafío poder crear dichos programas para brindar mayores oportunidades a los educandos que forman parte de estos colectivos históricamente excluidos y marginados socialmente.

El docente debe ser comprometido, presentar una actitud positiva y desarrollar la planificación, mediación, y evaluación formativa. La empatía, capacidad de escucha, motivación y canalizar las situaciones difíciles en el aula de clases complementan los aspectos didácticos, y pedagógicos en el aprendizaje. 
En la educación superior, se requiere un educador sensible y que sea consciente para aceptar y entender las diferencias de los estudiantes, puesto que, estas acciones permitirán realizar cambios significativos en los espacios de aprendizaje que forman parte de la enseñanza en este nivel. La democratización de los salones de clase en la educación, es producto de la cohesión y el respeto de los estudiantes, y el profesorado. Por esta razón, es vital que se construya esta relación reciproca diariamente.

La atención a la diversidad es un hecho adherido al proceso de formación inicial y permanente del profesorado universitario. Por tanto, solicita la participación activa de estudiantes y docentes para generar mejores oportunidades en la educación superior con el fin de evitar cualquier forma de discriminación y exclusión en contra de la colectividad de los educandos que manifiestan un sin número de características muy particulares. La creación de programas de formación de profesores es un factor clave para la generación de competencias básicas y genéricas para abordar a los estudiantes de diferentes colectivos sociales. Por esta razón, surge una propuesta de diez competencias básicas y transversales que pueden desarrollarse mediante los programas de profesionalización docente en los centros de enseñanza superior.

La formación inicial del profesorado, carece de un modelo coherente debido a que no ofrece respuestas convincentes para el abordaje a la diversidad en todas sus expresiones. Esta temática en los procesos formativos docentes se presenta a través de asignaturas optativas, lo que indica que tiene una mínima importancia dentro de las mallas curriculares establecidas. Esta etapa de preparación es vital para los futuros educadores, dado que, para brindar respuestas inmediatas a todos los colectivos que participan de manera cotidiana en los centros de educación superior deben promoverse aspectos actitudinales, creencias y desarrollar múltiples concepciones en base a la educación.

El modelo económico neoliberal que impera en distintos países latinoamericanos, es un factor que condiciona y desvaloriza cada vez más la profesión docente. De este modo, la universidad ha sufrido una serie de cambios en su estructura y funcionamiento. El aumento de exigencias que recaen sobre los académicos repercute de manera directa en la práctica educativa. Sin embargo, en el ejercicio educativo se han de integrar mecanismos que permitan la participación, fomenten el pluralismo y promuevan la inclusión en los espacios de aprendizaje.

Para terminar, la revisión presentada da cuenta de la necesidad de promover en América Latina nuevos estudios sobre la atención a la diversidad, para comprenderla desde múltiples perspectivas. Es necesario impulsar iniciativas que permitan consolidar la formación inicial y permanente del profesorado, en la búsqueda por democratizar las instituciones de enseñanza superior y posibilitar la participación directa de diversos grupos sociales en los espacios educativos universitarios.

\section{Agradecimientos}

Al Programa de Financiamiento de Movilidades y Becas Internacionales para el Relevo Docente de la Universidad Nacional Autónoma de Honduras (UNAH).

Al Dr. Ilich Silva Peña por sus enormes aportes para el desarrollo de este trabajo. 


\section{Referencias}

Aguaded, E. M., de la Rubia, P. y González, E. (2013). La importancia de la formación del profesorado en competencias interculturales. Profesorado. Revista de Currículum y Formación del Profesorado, $17(1)$, 339-365.

Álvarez, Q. (2018). Pedagogía sistémica e interculturalidad: Claves para construir un aula inclusiva. Revista Lusófona de Educação, 37,165-179.

Álvarez-Pérez, P. R. y López-Aguilar, D. (2015). Atención del profesorado universitario a estudiantes con necesidades educativas específicas. Educación y Educadores, 18(2), 193-208. https://dx.doi.org/10.5294/edu.2015.18.2.1

Amaro, M. C., Méndez, J. M. y Mendoza, F. (2014). Un estudio de las características profesionales del docente universitario para atender a la diversidad. Revista Latinoamericana de Educación Inclusiva, 8(2), 199-216.

Arnaiz, P. (2003). Multiculturalidad y diversidad en las aulas. Indivisa. Boletín de Estudios e Investigación, 4, 9-30.

Barrón Tirado, M. C. (2009). Docencia universitaria y competencias didácticas. Perfiles Educativos, 31(125), 76-87.

Booth, T. y Ainscow, M. (2015). Guía para la educación inclusiva. Desarrollando el aprendizaje y la participación en los centros escolares. Madrid: OEI, FUHEM.

Cañedo, T. J. y Figueroa, A. E. (2013). La práctica docente en educación superior: Una mirada hacia su complejidad. Sinéctica, 41, 2-18.

Cárdenas, T. J. (2012). Atención a la diversidad en el aula. Visión Educativa IUNAES, 12(5), 6371.

Danielson, C. (2011). Competencias docentes, desarrollo, apoyo y evaluación. PREAL, 51, 24-65.

Díez, E. y Sánchez, S. (2015). Diseño universal para el aprendizaje como metodología docente para atender a la diversidad en la universidad. Aula abierta, 43(2), 87-93. https://doi.org/10.1016/j.aula.2014.12.002

Domínguez, J. y Vázquez, E. (2015). Atención a la diversidad: Análisis de la formación permanente del profesorado en Galicia. Revista Nacional e Internacional de Educación Inclusiva, 8(2), 139-152.

Duran, S. y Parra, M. (2014). Diversidad Cultural para promover el desarrollo de habilidades sociales en educación superior. Cultura, Educación y Sociedad, 5(1), 55-67.

Eslava, M. D., de León, C. y González, I. (2015). La formación en competencias transversales para trabajar en entornos educativos inclusivos. Revista de Educación Inclusiva, 8(2), 58-76.

Fernández Batanero, J. M. (2012). Capacidades y competencias docentes para la inclusión del alumnado en la educación superior. Revista de la Educación Superior, 41(162), 9-24.

Fernández Batanero, J. M. (2013). Competencias docentes y educación inclusiva. Revista Electrónica de Investigación Educativa, 15(2), 82-99.

Ferrada, D. (2017). Formación docente para la diversidad: Propuestas desde la región del Biobío, Chile. Revista Mexicana de Investigación Educativa, 22(74), 783-811.

Freire, P. (1993). Cartas a quien pretende enseñar. Buenos Aires: Siglo Veintiuno Editores.

García, L. y Arroyo, M. (2014). La formación del profesorado en educación intercultural: Un repaso sobre su formación inicial y permanente. Revista de Educación Inclusiva, 7(2), 127142. 
García, I., Romero, S., Aguilar, L., Lomelí, K. y Rodríguez, D. (2013). Terminología internacional sobre la educación inclusiva. Actualidades Investigativas en Educación, 13(1), 1-29. https://doi.org/10.15517/aie.v13i1.11712

García, J. y Jáuregui, P. (2014). Interculturalidad y educación superior en México. Panorama del estado actual. Revista Latinoamericana de Educación Inclusiva, 8(1), 129-141.

Guzmán Marín, F. (2018). Los retos de la educación intercultural en el siglo XXI. Revista Latinoamericana de Educación Inclusiva, 12(1), 199-212. https://dx.doi.org/10.4067/S071873782018000100199

Herrera-Seda, C. M., Pérez-Salas, C. P. y Echeita, G. (2016). Teorías implícitas y prácticas de enseñanza que promueven la inclusión educativa en la universidad: Instrumentos y antecedentes para la reflexión y discusión. Formación Universitaria, 9(5), 49-64. https://doi.org/10.4067/SO7 18-50062016000500006

Jiménez, F. y Fardella, C. (2015). Diversidad y rol de la escuela: Discursos del profesorado en contextos educativos multiculturales en clave migratoria. Revista Mexicana de Investigación Educativa, 20(65), 419-441.

Larraín, A. M. y González, L. E. (2005, julio). Formación universitaria por competencias. Comunicación presentada en el Seminario internacional CINDA. Currículo universitario basado en competencias. Universidad del Norte, Colombia.

Le Boterf, G. (2001). Ingeniería de las competencias. Barcelona: Gestión 2000.

López-Melero, M. (2012). La escuela inclusiva: Una oportunidad para humanizarnos. Revista Interuniversitaria de Formación del Profesorado, 26(2), 131-160.

Martxueta, A. y Etxeberria, J. (2014). Claves para atender la diversidad afectivo-sexual en el contexto educativo desde un enfoque global escolar. Revista Española de Orientación y Psicopedagogía, 25(3), 121-128. https://doi.org/10.5944/reop.vol.25.num.3.2014.13862

Mas, O. y Olmos, P. (2016). El profesor universitario en el espacio europeo de educación superior: La autopercepción de sus competencias docentes actuales y orientaciones para su formación pedagógica. Revista Mexicana de Investigación Educativa, 21(69), 437-470.

Matus, L. G. (2013). La construcción de una identidad docente. ¿Un desafío para la política educativa? Revista Exitus, 3(1), 75-87.

Murillo, F. J., Krichesky, G., Castro, A. y Hernández, R. (2010). Liderazgo para la inclusión escolar y la justicia social. Aportaciones de la investigación. Revista Latinoamericana de Educación Inclusiva, 4(1), 169-186.

Olmos, A., Romo, M. R. y Arias, L. M. (2016). Reflexiones docentes sobre inclusión educativa: Relatos de experiencia pedagógica sobre la diversidad universitaria. Revista Latinoamericana de Educación Inclusiva, 10(1), 229-243. https://dx.doi.org/10.4067/S071873782016000100012

Parrilla, A. (1999). Más allá del conocimiento intelectual sobre la diversidad. Profesorado, Revista de Currículum y Formación del Profesorado, 3(2), 1-16.

Paz Maldonado, E. (2018a). Situación actual de la atención a la diversidad en la educación superior de Honduras. Revista Actualidades Investigativas en Educación, 18(3), 1-32. https://doi.org/10.15517/aie.v18i3.34148

Paz Maldonado, E. (2018b). La formación del profesorado universitario para la atención a la diversidad en la educación superior. Revista de Investigación Educativa de la REDIECH, 9(16), 67-82.

Perrenoud, P. H. (2013). Construire competenze a partire della scuola. Roma: Anicia. 
Rey, B. (1999). De las competencias transversales a una pedagogía de la intención. Recuperado de http://www.philosophia.cl/biblioteca.html

Rivadeneira, E. (2017). Competencias didácticas-pedagógicas del docente, en la transformación del estudiante universitario. Orbis. Revista Científica Ciencias Humanas, 13(37), 41-55.

Rodríguez, A., Álvarez, E. y García-Ruiz, R. (2014). La atención a la diversidad en la universidad: El valor de las actitudes. Revista Española de Orientación y Psicopedagogía, 25(1), 44-61. https://doi.org/10.5944/reop.vol.25.num.1.2014.12012

Rosales López, C. (2012). Diversidad y convivencia en los centros escolares. Una práctica en la formación inicial del futuro educador. Revista de Educación Inclusiva, 5(2), 75-92.

Salas Guzmán, N. y Salas Guzmán, M. (2016). Tiza de colores: Hacia la enseñanza de la inclusión sobre diversidad sexual en la formación inicial docente. Revista Latinoamericana de Educación Inclusiva, 10(2), 73-91. https://doi.org/10.4067/So718-73782016000200006

Salinas, J., de Benito, B. y Lizana, A. (2014). Competencias docentes para los nuevos escenarios de aprendizaje. Revista Interuniversitaria de Formación del Profesorado, 28(1), 145-163.

Santos, M. A., Cernadas. F. X. y Lorenzo, M. M. (2014). La inclusión educativa de la inmigración y la formación intercultural del profesorado. Revista Electrónica Interuniversitaria de Formación del Profesorado, 17(2), 123-137. https://doi.org/10.6018/reifop.17.2.196931

Schilling, C. (2018). Formación docente. Experiencias de prácticas pedagógicas y trabajo comunitario como espacio de reconocimiento. En D. Ferrada (Ed.), Reflexiones y experiencias educativas desde las comunidades (pp. 149-172). Talca: Ediciones UCM.

Silva-Peña, I. (2017). Formación docente para la justicia social en un Chile desigual. En I. SilvaPeña, J. Diniz-Pereira. y K. Zeichner (Eds.), Justicia social. La dimensión olvidada de la formación docente (pp. 121-145). Santiago: Mutante Editores.

Tobar, A. O. (2017). Índice de competencias TIC en docentes de educación superior. Campus Virtuales, 6(2), 113-125.

Torres, A. D., Badillo, M., Valentín, N. O. y Ramírez, E. T. (2014). Las competencias docentes: El desafío de la educación superior. Innovación Educativa, 14(66), 129-145.

Valcárcel, N. I., Quintana, L. A. L. y Oramas, R. G. (2014). Las competencias del profesor universitario. Revista Ciencias Pedagógicas e Innovación, 2(2), 34-39.

Vélez Calvo, X., Tárraga Mínguez, R., Fernández Andrés, M. I. y Sanz Cervera, P. (2016). Formación inicial de maestros en educación inclusiva: una comparación entre Ecuador y España. Revista Nacional e Internacional de Educación Inclusiva, 9(3), 75-94.

Veloquio, G. G. (2016). La formación permanente de los docentes, ante el desafío de atender a la diversidad educativa. Revista de Educación Inclusiva, 9(2), 144-154.

Villarroel, V. y Bruna, D. (2014). Reflexiones en torno a las competencias genéricas en educación superior: Un desafío pendiente. Psicoperspectivas, 13(1), 23-34. https://doi.org/10.5027/psicoperspectivas-Vol13-Issue1-fulltext-335

Zabalza, M. (2003). Competencias docentes del profesorado universitario: Calidad y desarrollo profesional. Madrid: Narcea.

Zabalza, M. (2009). Ser profesor universitario hoy. La Cuestión Universitaria, 5, 69-81.

Zabalza, M. (2012). Las competencias en la formación del profesorado: De la teoría a las propuestas prácticas. Tendencias Pedagógicas, 20, 5-32.

Zeichner, K. (2017). Formación de profesores para la justicia social en tiempos de rendición de cuentas, incertidumbre y crecientes desigualdades. En I. Silva-Peña, J. Diniz-Pereira y K. 
Zeichner (Eds.), Justicia social. La dimensión olvidada de la formación docente (pp. 15-35). Santiago: Mutante Editores.

\section{Breve CV del autor}

\section{Eddy Paz Maldonado}

Profesor Escuela de Pedagogía y Ciencias de la Educación, Universidad Nacional Autónoma de Honduras (UNAH). Magister en Educación Especial y Psicopedagogía Universidad Católica del Maule, Chile. Licenciado en Pedagogía y Ciencias de la Educación con Orientación en Administración y Planeamiento de la Educación, UNAH. Líneas de investigación vinculadas a: Inclusión Educativa, Colectivos Vulnerables y Atención a la Diversidad en la Enseñanza Superior. ORCID ID: https://orcid.org/oooo0002-2324-8813. Email: eddy.paz@unah.edu.hn 